\title{
BERYLLIUM ABUNDANCES OF F DWARFS
}

\author{
Kent G. Budge \\ Ann Merchant Boesgaard \\ Palomar Observatory and Department of Astronomy \\ California Institute of Technology \\ John Varsik \\ Institute for Astronomy \\ University of Hawaii
}

\begin{abstract}
We have obtained spectra near $3130 \AA$ of $23 \mathrm{~F}$ and $\mathrm{G}$ main-sequence stars at $0.05 \AA \mathrm{px}^{-1}$. We have made preliminary estimates of the equivalent widths for the Be II doublet at 3130.4 and $3131.1 \AA$ by means of a comparison procedure that matches each star against a standard star of similar spectral type with known $\mathrm{Be}$ abundance. It appears that stars that are deficient in $\mathrm{Be}$ are very uncommon. The halo dwarf, HD 76932, clearly has Be.
\end{abstract}

\section{INTRODUCTION}

The beryllium abundance of a main-sequence star is an indication of the depth and extent of convective mixing, since $\mathrm{Be}$ is destroyed at moderately low temperatures $\left(\sim 3.5 \times 10^{6} \mathrm{~K}\right)$. In particular, one might expect a subset of the known Li-deficient stars to be Be-deficient as well. The cosmic Be abundance is also of interest, since $\mathrm{Be}$ is formed exclusively by spallation and is therefore a measure of cosmic-ray activity during the lifetime of the galaxy.

\section{OBSERVATIONS}

We have obtained spectra for a number of $F$ main-sequence stars from various populations, including stars in the Hyades Li gap (Boesgaard and Trippico 1986) and the halo dwarf HD 76932. Observations were made at CFHT on the nights of October 10 and December 10-11, 1986. Spectra were obtained at the coudé focus using the ultraviolet optical train and a TI CCD as detector. The dispersion was $.05 \AA /$ pixel and the signal-to-noise ratio was typically $\sim 100$ as opposed to $\sim 20$ for IUE. Although progress has been made in increasing the blue sensitivity of CCD detectors, exposures of $\sim 2$ hours were required to obtain this signal-to-noise ratio for the fainter Hyades stars. 


\section{REDUCTION OF DATA}

The spectra have been flat-fielded and dark-subtracted and cosmic ray signatures removed by an interpolation process from adjoining pixels. The spectral region near the Be doublet is heavily line-blanketed, making it difficult to determine the true continuum level. This line-blanketing also results in numerous blends, which are further accentuated by the rapid rotation of many stars of interest in this temperature range.

To solve these problems, a "differential spectroscopy" procedure is being developed. This procedure includes the following steps: First, a cross-correlation algorithm is used to match the wavelength scale of the object spectrum to a standard spectrum. The object spectrum is then multiplied by a low-order polynomial (usually linear) selected by a least-squares algorithm to give the best fit between the object and standard spectra in the region immediately around the Be doublet. Finally, the standard spectrum is subtracted from the object spectrum. The resulting difference spectrum shows any differences between the object and standard spectra; furthermore, if the continuum level is known for the standard, the equivalent width of the $\mathrm{Be}$ doublet may be obtained. This procedure is adapted to rapidly-rotating stars by convolving the standard spectrum with a rotation profile of the correct width.

Figure 1 shows the results of this procedure as applied to the object star $\iota$ Psc (heavy line at top) with the standard star $\gamma^{1}$ Del (light line at top). The difference spectrum, showing the signature of $\mathrm{Be}$, appears at the botton of the figure.

This procedure reduces the problems with close blends and, assuming the continuum level has been carefully determined for the standard stars, avoids the necessity of determining the continuum level for the object stars. However, it requires that standard stars of known Be abundance be available. We have selected Procyon ( $\alpha$ CMa, F5IV-V) as our primary standard. Although this star has spectral peculiarities arising from its location slightly above the main sequence, its spectrum shows no detectable $\mathrm{Be}$, a result consistent with a probable history of mass transfer between it and its white dwarf companion. In addition, Procyon has a very small value of $v \sin i$. Our secondary standards include $\gamma^{1} \operatorname{Del}($ F7V), $\xi$ Peg (F7V), and $\chi^{1}$ Ori (G0V), all of which have small values of $v$ sin $i$. All of these stars have normal Be abundance except for $\gamma^{1}$ Del, which appears to be somewhat deficient in $\mathrm{Be}$.

\section{RESULTS}

Our preliminary results indicate that Be deficiency is a much rarer phenomenon than $\mathrm{Li}$ deficiency (Boesgaard 1976). This is not particularly surprising when one considers that $\mathrm{Be}$ is destroyed at temperatures of $\sim 3.5 \times 10^{6} \mathrm{~K}$ while $\mathrm{Li}$ is destroyed at only $\sim 2.5 \times 10^{6} \mathrm{~K}$. We find no evidence for Be deficiency in the Hyades Li-gap stars, although the rapid rotation of these stars makes the measurement particulary uncertain. We also find that HD 76932, the halo dwarf, is definitely overabundant in $\mathrm{Be}$, with a near-solar abundance of this element despite an overall low metallicity (Figure 2). This suggests that much of the galactic Be was formed early, but measurements are needed for more halo dwarfs.

This work was supported by NSF grants AST-8216192 and RII-8521715 and a Guggenheim Fellowship to AMB and by an NSF Graduate Fellowship to KGB. 


\section{REFERENCES}

Boesgaard, A.M. 1976, Astrophys. J., 210, 466.

Boesgaard, A.M., and Trippico, M.J. 1986, Astrophys. J., 302, L49.

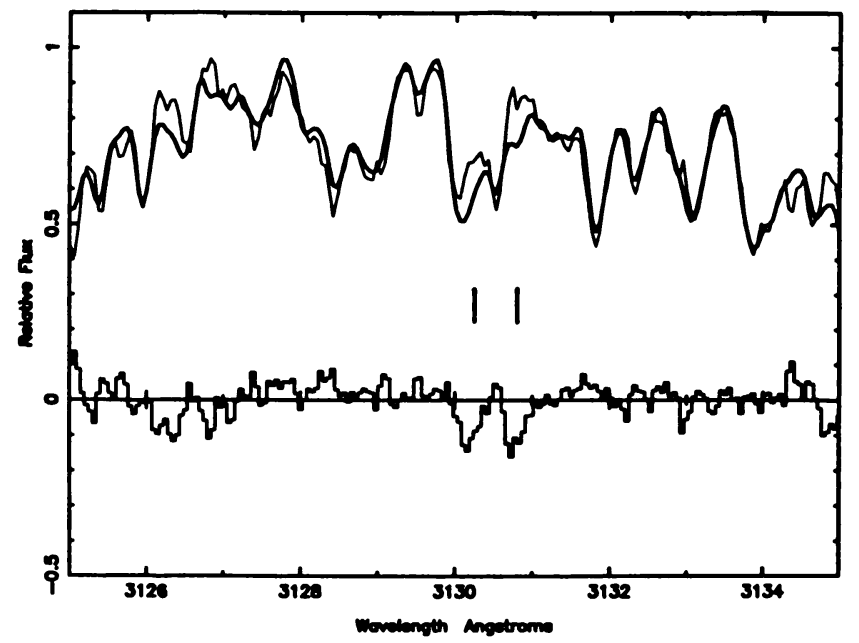

Figure 1. $\iota$ Psc vs. $\gamma^{1}$ Del. The Be signature is seen clearly in the lower spectrum.

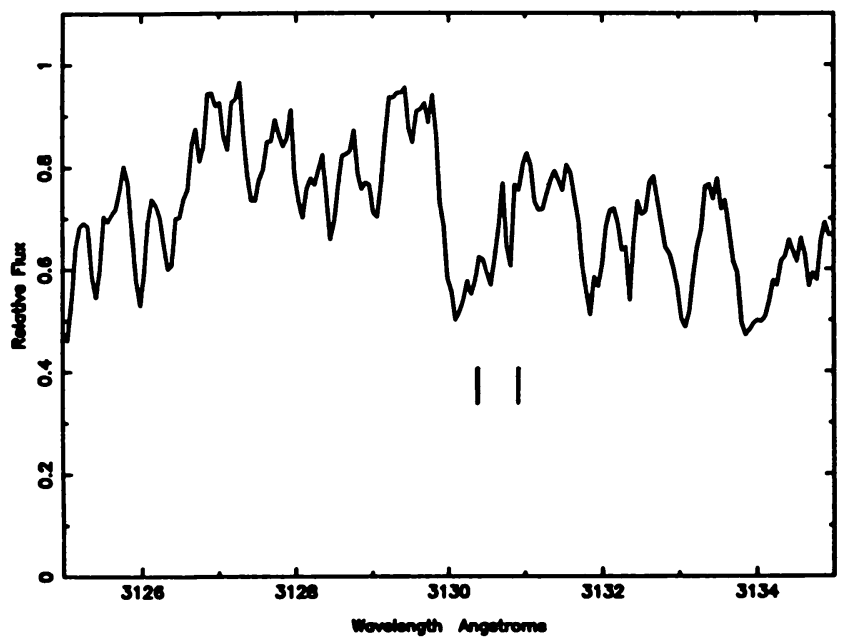

Figure 2. The halo dwarf HD 76932 
DISCUSSION

VAUCLAIR Can you give approximate values of the beryllium abundances in stars on each side of the lithium gap in the Byades, and the uncertainty on these values?

BUDEE Our numbers are very rough at this stage, I wouldn't claim better than a factor of two uncertainty. However, Be/H in VBI4 is around 1.2x10-11, VB37 is possibly somewhat depleted, perhaps Be/Hzaxx1012. Other Hyades stars all fall in this range.

SODERBLOM Have you Be observations of other halo owarfs to see how ubiquitous their high Be is?

BUDGE Unfortunately, no. We do have some Be observations of the old disk, which show roughly solar Be, but no other halo dwarfs. 